\title{
Exact Controllability for Hilfer Fractional Differential Inclusions Involving Nonlocal Initial Conditions
}

\author{
Jun Du $\mathbb{D},,^{1,2}$ Wei Jiang $\mathbb{D}^{1},{ }^{1}$ Denghao Pang, ${ }^{1}$ and Azmat Ullah Khan Niazi $\mathbb{D}^{1}$ \\ ${ }^{1}$ School of Mathematical Sciences, Anhui University, Hefei 230601, China \\ ${ }^{2}$ Department of Applied Mathematics, Huainan Normal University, Huainan 232038, China
}

Correspondence should be addressed to Jun Du; djwlm@163.com and Wei Jiang; jiangwei@ahu.edu.cn

Received 27 March 2018; Accepted 11 July 2018; Published 19 August 2018

Academic Editor: Dimitri Volchenkov

Copyright ( 2018 Jun Du et al. This is an open access article distributed under the Creative Commons Attribution License, which permits unrestricted use, distribution, and reproduction in any medium, provided the original work is properly cited.

\begin{abstract}
The exact controllability results for Hilfer fractional differential inclusions involving nonlocal initial conditions are presented and proved. By means of the multivalued analysis, measure of noncompactness method, fractional calculus combined with the generalized Mönch fixed point theorem, we derive some sufficient conditions to ensure the controllability for the nonlocal Hilfer fractional differential system. The results are new and generalize the existing results. Finally, we talk about an example to interpret the applications of our abstract results.
\end{abstract}

\section{Introduction}

Fractional calculus generalizes the standard integer calculus to arbitrary order. It provides a valuable tool for the description of memory and hereditary properties of diversified materials and processes. In the past twenty years, the subject of the fractional calculus is picking up considerable popularity and importance. We can refer to the monographs of Diethelm and Freed [1], Kilbas et al. [2], Miller and Ross [3], Podlubny [4], and Zhou [5]. Fractional differential equations and inclusions involving Caputo derivative or Riemann-Liouville derivative have obtained more and more results (see [6-15]). Recently, Hilfer [16] initiated an extended Riemann-Liouville fractional derivative, named Hilfer fractional derivative, which interpolates Caputo fractional derivative and Riemann-Liouville fractional derivative. This operator appeared in the theoretical simulation of dielectric relaxation in glass forming materials. Hilfer et al. [17] initially presented linear differential equations with the new Hilfer fractional derivative and applied operational calculus to solve such generalized fractional differential equations. Subsequently, Furati et al. [18] and $\mathrm{Gu}$ and Trujillo [19] generalized to consider nonlinear problems and proved the existence, nonexistence, and stability results for initial value problems of nonlinear fractional differential equations with Hilfer fractional derivative in a suitable weighted space of continuous functions.

Control theory is an interdisciplinary branch of engineering and mathematics that deals with influence behavior of dynamical systems. Controllability is one of the fundamental concepts in mathematical control theory, it means that it is possible to steer a dynamical system from an arbitrary initial state to arbitrary final state using the set of admissible controls. Recently, the controllability conditions for various linear and nonlinear integer or fractional order systems have been considered in many papers by using different methods [20-33] and the references. There have also been some results [20-24, 32, 33] about the investigations of the exact controllability of systems represented by nonlinear evolution equations in infinite dimensional space. But when the semigroup or the control action operator B is compact, then the controllability operator is also compact and the applications of exact controllability results is just restricted to the finite dimensional space [20]. Therefore, we investigate the exact controllability of the fractional evolution systems only involving noncompact semigroups.

The nonlocal initial problems have been initially proposed by Byszewski et al. [34, 35] to generalize the study of the canonical initial problem, comes from physical science. For instance, it used to determine the unknown physical 
parameters in some inverse heat condition problems. It has been found that the nonlocal initial condition is more exact to describe the nature phenomena than the classical initial condition, since more data is taken into account, therefore abating the negative influences induced by a possible inaccurate single estimation taken at the start time. For more discussion on this type of differential equations and inclusions, we can see papers [36-42] and references given therein.

Boucherif and Precup [36] proved the existence for mild solutions to the following nonlocal initial problem for first-order evolution equations using Schaefer fixed point theorem:

$$
\begin{aligned}
x^{\prime}(t)+A x(t) & =f(t, x(t)), t \in J, \\
x(0)+\sum_{k=1}^{m} a_{k} x\left(t_{k}\right) & =0,0<t_{1}<t_{2}<\cdots<t_{m}<b,
\end{aligned}
$$

where $A: D(A) \subseteq X \rightarrow X$ is the infinitesimal generator of a $C_{0}$-semigroup $\{T(t)\}_{t \geq 0}$ on a Banach space $X$ and $f: J \times X \rightarrow X$ is a known function.

Liang and Yang [33] concerned the controllability for the following fractional integrodifferential evolution equations involving nonlocal conditions using the Mönch fixed point theorem:

$$
\begin{aligned}
D^{q} x(t)+A x(t) & =f(t, x(t), G x(t))+B u(t), t \in J, \\
x(0) & =\sum_{k=1}^{m} c_{k} x\left(t_{k}\right), 0<t_{1}<t_{2}<\cdots<t_{m}<b,
\end{aligned}
$$

where $D^{q}$ is the Caputo derivative of order $q \in(0,1)$, $-A: D(A) \subseteq X \rightarrow X$ is the infinitesimal generator of $C_{0}$-semigroup $\{T(t)\}_{t \geq 0}$ of uniformly bounded linear operator, the control function $u$ is known in $L^{2}(J, U)$; $U$ is a Banach space, $B$ is a linear bounded operator from $U$ to $X ; f$ is a known function and $G x(t)=\int_{0}^{t} K(t, s) x(s) d s$ is a Volterra integral operator.

$\mathrm{Du}$ et al. [43] generalized the results of [33] and gave the controllability for a new class of fractional neutral integrodifferential evolution equations with infinite delay and nonlocal conditions using Mönch fixed point theorem. However, it should be emphasized that to the best of our knowledge, the exact controllability of Hilfer fractional differential system has not been investigated yet. Motivated by $[19,30,33,36,43]$, in this paper, we concern the controllability of the following fractional differential inclusions involving a more general fractional derivative with nonlocal initial conditions:

$$
\begin{array}{r}
D_{0^{+}}^{p, q} x(t) \in A x(t)+F(t, x(t))+B u(t), t \in(0, a], \\
I_{0^{+}}^{(1-q)(1-p)} x\left(0^{+}\right)=\sum_{i=1}^{n} a_{i} x\left(t_{i}\right), 0<t_{1}<t_{2}<\cdots<t_{n}<a,
\end{array}
$$

where $D_{0^{+}}^{p, q}$ is the Hilfer fractional derivative of order $p$ ( $p$ obeys $1 / 2<p \leq 1$ ) and type $q$ ( $q$ obeys $0 \leq q \leq 1$ ) which will be given in Section 2; $E$ is separable and $A$ is bounded, so $S(\cdot)$ is a uniformly continuous semigroup and $S(t)=e^{A t}$. The nonlinear term $F: J \times E \rightarrow 2^{E} \backslash\{\varnothing\}$ is multivalued function. Let $J=[0, a], J^{\prime}=(0, a], a>0$ are two finite intervals of $\mathbb{R}$; $a_{i} \in \mathbb{R}, a_{i} \neq 0(i=1,2, \ldots, n), n \in N$. The control function $u$ takes values in $L^{2}(J, U)$, with $U$ as a Banach space; $B$ is a linear bounded operator from $U$ to $E$.

In this paper, by means of a concrete nonlocal function, we do not have to suppose the compactness and Lipschitz conditions on the nonlocal function but only assume that $a_{i}$ $(i=1,2, \ldots, n)$ satisfy the hypothesis (H0) (see Section 3 ). Furthermore, the proofs of our main results are based on fractional calculus theory, the multivalued analysis, measure of noncompactness method, in addition to the O'ReganPrecup fixed point theorem, which is an extension of the Mönch fixed point theorem.

\section{Preliminaries and Notations}

Let $C\left(J^{\prime}, E\right)$ and $C(J, E)$ denote the space of $E$-valued continuous functions from $J^{\prime}$ to $E$ and from $J$ to $E$, respectively; Let $r=p+q-p q$.

Define $Y=\left\{x \in C\left(J^{\prime}, E\right): \lim _{t \rightarrow 0^{+}} t^{1-r} x(t)\right.$ exists and is finite $\}$, involving the norm $\|\cdot\|_{Y}$ defined by $\|x\|_{Y}=$ $\sup _{t \in J}\left\{t^{1-r}|x(t)|\right\}$. Then, $Y$ is a Banach space. We also note that

(1) When $r=1$, then $Y=C(J, E)$ and $\|\cdot\|_{Y}=\|\cdot\|$;

(2) Let $x(t)=t^{r-1} y(t)$ for $t \in J^{\prime}, x \in Y$ if and only if $y \in C(J, E)$, and $\|x\|_{Y}=\|y\|$.

For $\gamma>0$, define $B_{\gamma}^{Y}\left(J^{\prime}\right)=\left\{x \in Y:\|x\|_{Y} \leq \gamma\right\}$. Thus $B_{\gamma}^{Y}$ is a bounded closed and convex subset of $Y$.

Let $B_{\gamma}(J)=\{y \in C(J, E):\|y\| \leq \gamma\}$, Then $B_{\gamma}$ is a closed ball of the space $C(J, E)$ with the radius $\gamma$ and center at 0 . And $B_{\gamma}$ is also a bounded closed and convex subset of $C(J, E)$.

Next, we list some definitions and properties in fractional calculus, multivalued analysis, semigroup theory, and measure of noncompactness.

The following definitions concerning fractional calculus can be found in the books $[2-4,16]$.

Definition 1. The fractional integral for function $f$ from lower limit 0 and order $\alpha$ can be expressed by

$$
I_{0^{+}}^{\alpha} f(t)=\frac{1}{\Gamma(\alpha)} \int_{0}^{t} \frac{f(s)}{(t-s)^{1-\alpha}} d s, \alpha>0, t>0
$$

where $\Gamma$ is the gamma function, and right side of upper equality is point-wise defined on $[0,+\infty)$. 
Definition 2. The Riemann-Liouville derivative of order $\alpha$ with the lower limit 0 for function $f:[0,+\infty) \rightarrow \mathbb{R}$ can be expressed by

$$
\begin{aligned}
{ }^{R-L} D_{0^{+}}^{\alpha} f(t)= & \frac{1}{\Gamma(n-\alpha)} \frac{d^{n}}{d t^{n}} \\
& \cdot \int_{0}^{t} \frac{f(s)}{(t-s)^{\alpha+1-n}} d s, t>0,0 \leq n-1<\alpha<n .
\end{aligned}
$$

Definition 3. The Caputo derivative of order $\alpha$ for function $f:[0,+\infty) \rightarrow \mathbb{R}$ can be denoted by

$$
{ }^{C} D_{0^{+}}^{\alpha} f(t)={ }^{R-L} D^{\alpha}\left(f(t)-\sum_{k=0}^{n-1} \frac{t^{k}}{k !} f^{(k)}(0)\right), t>0,0 \leq n-1<\alpha<n .
$$

Definition 4. The left Hilfer derivative of order $0<p \leq 1$ and type $0 \leq q \leq 1$ of function $f$ is defined by

$$
D_{0^{+}}^{p, q} f(t)=\left(I_{0^{+}}^{q(1-p)} D\left(I_{0^{+}}^{(1-q)(1-p)} f\right)\right)(t)
$$

where $D:=d / d t$

\section{Remark 1.}

(i) The operator $D_{0^{+}}^{p, q}$ can be written as

$$
\begin{aligned}
D_{0^{+}}^{p, q} f(t) & =\left(I_{0^{+}}^{q(1-p)} D\left(I_{0^{+}}^{1-r} f\right)\right)(t) \\
& =\left(I_{0^{+}}^{q(1-p)}\left(D^{r} f\right)\right)(t), r=p+q-p q .
\end{aligned}
$$

(ii) When $q=0$ and $0<p \leq 1$, the Hilfer fractional derivative coincides with the Riemann-Liouville derivative:

$$
D_{0^{+}}^{p, 0} f(t)=\frac{d}{d t}\left(I_{0^{+}}^{1-p} f\right)(t)={ }^{R-L} D_{0^{+}}^{p} f(t) .
$$

(iii) When $q=1$ and $0<p \leq 1$, the Hilfer fractional derivative coincides with the Caputo derivative:

$$
D_{0^{+}}^{p, 1} f(t)=I_{0^{+}}^{1-p} \frac{d}{d t} f(t)={ }^{C} D_{0^{+}}^{p} f(t)
$$

Let $\mathscr{P}(E)$ be the set of all nonempty subsets of $E$. We will use the following notations:

$$
\begin{aligned}
& \mathscr{P}_{c l}(E):=\{Y \in \mathscr{P}(E) \mid Y \text { is closed }\}, \\
& \mathscr{P}_{b}(E):=\{Y \in \mathscr{P}(E) \mid Y \text { is bounded }\}, \\
& \mathscr{P}_{c v}(E):=\{Y \in \mathscr{P}(E) \mid Y \text { is convex }\}, \\
& \mathscr{P}_{c p}(E):=\{Y \in \mathscr{P}(E) \mid Y \text { is compact }\} .
\end{aligned}
$$

\section{Remark 2.}

(i) A measurable function $u: J \rightarrow E$ is Bochner integrable if and only if $\|u\|$ is Lebesgue integrable.

(ii) A multivalued map $F: E \rightarrow 2^{E}$ is said to be convex valued (closed valued) if $F(u)$ is convex (closed) for all $u \in E$ is said to be bounded on bounded sets if $F(B)=\cup_{u \in B}$ is bounded in $E$ for all $B \in \mathscr{P}_{b}(E)$.

(iii) A multivalued map $F$ is said to be upper semicontinuous (u.s.c.) on $E$ if for each $u_{0} \in E$, the set $\mathrm{F}\left(u_{0}\right)$ is a nonempty closed subset of $E$, and if for each open subset $\Omega$ of $E$ containing $F\left(u_{0}\right)$, there exists an open neighborhood $\nabla$ of $u_{0}$ such that $F(\nabla) \subseteq \Omega$.

(iv) A multivalued map $F$ is said to be completely continuous if $F(B)$ is relatively compact for every $B \in \mathscr{P}_{b}(E)$. If the multivalued map $F$ is completely continuous with nonempty compact values, then $F$ is u.s.c. if and only if $F$ has a closed graph, that is, $u_{n} \rightarrow u, y_{n} \rightarrow y, y_{n} \in F\left(u_{0}\right)$ imply $y \in F(u)$. We say that $F$ has a fixed point if there is $u \in E$, such that $u \in F(u)$.

(v) A multivalued map $F: J \rightarrow \mathscr{P}_{c l}(E)$ is said to be measurable if for each $u \in E$, the function $y: J \rightarrow R$ defined by $y(t)=d(u, F(t))=\inf \{\|u-z\|, z \in F(t)\}$ is measurable.

Lemma 1 (see [44]). Let E be a Banach space. The multivalued map satisfies the following: for each $t \in J, F(t, \cdot): E \rightarrow$ $\mathscr{P}_{b, c l, c v}(E)$ is u.s.c.; for each $x \in E$, the function $F(\cdot, x): E \rightarrow$ $\mathscr{P}_{b, c l, c v}(E)$ is strongly measurable and the set $S_{F, x}=\left\{f \in L^{1}\right.$ $(J, E): f(t) \in F(t, x(t))$, for a.e. $t \in J\}$ is nonempty. Let $\Gamma$ be a linear continuous mapping from $L^{1}(J, E)$ to $C(J, E)$, then the operator

$$
\Gamma \circ S_{F}: C(J, E) \rightarrow \mathscr{P}_{b, c l, c v}(C(J, E)), x \mapsto\left(\Gamma \circ S_{F}\right)(x)=\Gamma\left(S_{F, x}\right)
$$

is a closed graph operator in $C(J, E) \times C(J, E)$.

Consider

$$
K_{p}(t)=p \int_{0}^{\infty} \theta \xi_{p}(\theta) S\left(t^{p} \theta\right) d \theta, t \geq 0
$$

where $\xi_{p}(\theta)=(1 / p) \theta^{-1-1 / 2} \omega_{p}\left(\theta^{-1 / 2}\right)$, and

$$
\omega_{p}(\theta)=\frac{1}{\pi} \sum_{n=1}^{\infty}(-1)^{n-1} \theta^{-p n-1} \frac{\Gamma(n p+1)}{n !} \sin (n \pi p), \theta \in(0, \infty),
$$

and $\xi_{p}$ is a probability density function defined on $(0, \infty)$, that is $\int_{0}^{\infty} \xi_{p}(\theta) d \theta=1$. 
Remark 3 (see [2]). As we all know from [2] that $K_{p}$ can be denoted by the Mittag-Leffler functions:

$$
K_{p}(t)=\sum_{i=0}^{\infty} \frac{A^{i} t^{p i}}{\Gamma(p i+p)}
$$

The following essential propositions can be found in the papers $[19,38]$.

Lemma 2. If $\|S(t)\| \leq N, t \in J$, then for each $x \in E$,

(i) $\left\|K_{p}(t) x\right\| \leq(N / \Gamma(p))\|x\|$;

(ii) $\left\|t^{p-1} K_{p}(t) x\right\| \leq\left(N t^{p-1} / \Gamma(p)\right)\|x\|$ and $\| I_{0^{+}}^{q(1-p)}\left(t^{p-1}\right.$ $\left.K_{p}(t)\right) x\left\|\leq\left(N t^{r-1} / \Gamma(r)\right)\right\| x \|$.

Lemma 3 (Example 2.1.3 [45]).

For each $G \subset C(J, E)$ and $t \in J$, define $G(t)=\{g(t)$ : $g \in G\}$. If $G$ is equicontinuous and bounded, then $\beta(G(t))$ is continuous on $J$ and

$$
\beta(G)=\max _{t \in J} \beta(G(t))
$$

Here, $\beta$ is the Hausdorff noncompact measure on $E$ defined on every bounded subset $U$ of Banach space E by

$$
\beta(U)=\inf \{\varepsilon>0, U \text { has a finite } \varepsilon-n e t \text { in } E\} .
$$

Lemma 4 (see Lemma 5 [46]). Let $G \subseteq L^{1}(J, E)$ be a countable subset with $\|g(t)\| \leq \varphi(t)$, for almost everywhere $t \in J$ and any $g \in G$, where $\varphi \in L^{1}\left(J, \mathbb{R}^{+}\right)$. Then

$$
\beta\left(\left\{\int_{J} g(t) d t: g \in G\right\}\right) \leq \int_{J} \beta(G(t)) d t .
$$

To end this section, we reintroduce the O'Regan-Precup fixed point theorem.

Lemma 5 (see Theorem 3.2 [47]). Let D be a subset of Banach space $E$ which is closed and convex. $\Omega$ is a relatively open subset of $D$, and $\mathscr{T}: \bar{\Omega} \rightarrow \mathscr{P}_{c v}(D)$. Suppose graph $(\mathscr{T})$ is closed, $\mathscr{T}$ maps compact sets into relatively compact sets, and that for some $x_{0} \in \Omega$, the following two conditions are satisfied:

$$
\left.\begin{array}{c}
Z \subseteq D, Z \subset \operatorname{conv}\left(\left\{x_{0}\right\} \cup \mathscr{T}(Z)\right) \\
\bar{Z}=\bar{G} \text { with } G \subseteq Z \text { countable }
\end{array}\right\} \Rightarrow \bar{Z} \text { compact },
$$

Then $\mathscr{T}$ has a fixed point.

\section{Controllability Results}

We first consider linear Hilfer fractional differential equations of the form

$$
\begin{aligned}
D_{0^{+}}^{p, q} x(t) & =A x(t)+h(t), t \in J^{\prime}, \\
I_{0^{+}}^{1-r} x\left(0^{+}\right) & =\sum_{i=1}^{n} a_{i} x\left(t_{i}\right),
\end{aligned}
$$

where $h \in C(J, E)$.

Assume that there exists the bounded operator $K: E \rightarrow E$ given by

$$
K:=\left[I-\sum_{i=1}^{n} a_{i} \Phi_{p, r}\left(A, t_{i}\right)\right]^{-1},
$$

where $\Phi_{p, r}(A, t)=I_{0^{+}}^{q(1-p)}\left(t^{p-1} K_{p}(t)\right)$.

By means of [48], we can present the sufficient conditions for the existence and boundedness of the operator $K$.

Lemma 6. If the hypothesis (HO) $\sum_{i=1}^{n}\left|a_{i}\right|<(\Gamma(r) / N) t_{1}{ }^{1-r}$ holds, the operator $K$ defined in (21) exists and is bounded.

Proof 1. From the hypothesis $(H 0)$, we have

$$
\left\|\sum_{i=1}^{n} a_{i} \Phi_{p, r}\left(A, t_{i}\right)\right\| \leq \frac{N}{\Gamma(r)} \sum_{i=1}^{n}\left|a_{i}\right| \cdot t_{i}^{r-1}<\frac{N t_{1}^{r-1}}{\Gamma(r)} \sum_{i=1}^{n}\left|a_{i}\right|<1 .
$$

By operator spectrum theorem, the operator $K:=$ $\left[I-\sum_{i=1}^{n} a_{i} \Phi_{p, r}\left(A, t_{i}\right)\right]^{-1}$ exists and is bounded. Furthermore, by Neumann expression, we get

$$
\begin{aligned}
\|K\| & \leq \sum_{n=0}^{\infty}\left\|\sum_{i=1}^{n} a_{i} \Phi_{p, r}\left(A, t_{i}\right)\right\|^{n}=\frac{1}{1-\left\|\sum_{i=1}^{n} a_{i} \Phi_{p, r}\left(A, t_{i}\right)\right\|} \\
& \leq \frac{1}{1-\left(N t_{1}^{r-1} / \Gamma(r)\right) \sum_{i=1}^{n}\left|a_{i}\right|} .
\end{aligned}
$$

Using Lemma 6 and [19], we give the following definition of mild solution for the Hilfer fractional system (20) involving nonlocal initial conditions.

Definition 5. A function $x \in C\left(J^{\prime}, X\right)$ is called a mild solution for the Hilfer fractional system (20) if it satisfies the following equation:

$$
\mathrm{x}(t)=\Phi_{p, r}(A, t) \sum_{i=1}^{n} a_{i} K\left(\bar{h}\left(t_{i}\right)\right)+\bar{h}(t), t \in J^{\prime},
$$

where $\bar{h}(t)=\int_{0}^{t} \Phi_{p, p}(A, t-s) h(s) d s$. 
Remark 4. By virtue of [19], a mild solution for Hilfer fractional evolution (20) with the initial condition is

$$
x(t)=\Phi_{p, r}(A, t) I_{0^{+}}^{1-r} x\left(0^{+}\right)+\int_{0}^{t} \Phi_{p, p}(A, t-s) h(s) d s .
$$

Specially,

$$
x\left(t_{i}\right)=\Phi_{p, r}\left(A, t_{i}\right) I_{0^{+}}^{1-r} x\left(0^{+}\right)+\int_{0}^{t_{i}} \Phi_{p, p}\left(A, t_{i}-s\right) h(s) d s .
$$

Using (20) and (26), we get

$$
\left[I-\sum_{i=1}^{n} a_{i} \Phi_{p, r}\left(A, t_{i}\right)\right] \cdot I_{0^{+}}^{1-r} x\left(0^{+}\right)=\sum_{i=1}^{n} a_{i} \int_{0}^{t_{i}} \Phi_{p, p}\left(A, t_{i}-s\right) h(s) d s .
$$

Since $I-\sum_{i=1}^{n} a_{i} \Phi_{p, r}\left(A, t_{i}\right)$ exists a bounded inverse operator which is denoted by $K$, so

$$
I_{0^{+}}^{1-r} x\left(0^{+}\right)=\sum_{i=1}^{n} a_{i} K \int_{0}^{t_{i}} \Phi_{p, p}\left(A, t_{i}-s\right) h(s) d s .
$$

And hence,

$$
x(t)=\Phi_{p, r}(A, t) \sum_{i=1}^{n} a_{i} K\left(\bar{h}\left(t_{i}\right)\right)+\bar{h}(t), t \in J^{\prime},
$$

it is indeed (24).

Using Definition 5, we give a new definition of the mild solution for the Hilfer fractional nonlocal differential inclusions (3) as follows:

Definition 6. A function $x \in C\left(J^{\prime}, E\right)$ is called a mild solution of the Hilfer fractional nonlocal differential inclusions (3) if for any $u \in L^{2}(J, U)$, the following integral equation is satisfied:

$$
x(t)=\Phi_{p, r}(A, t) \sum_{i=1}^{n} a_{i} K\left(\bar{f}\left(t_{i}\right)\right)+\bar{f}(t), t \in J^{\prime},
$$

where $\bar{f}(t)=\int_{0}^{t} \Phi_{p, p}(A, t-s)(B u(s)+f(s)) d s, \quad \Phi_{p, p}(A, t)=$ $t^{p-1} K_{p}(t)$ and $f \in S_{F, x}$.

To present and prove the main results of this paper, we enumerate the following hypotheses:

(H1) $E$ is a separable Banach space, $A$ is bounded, hence $S(\cdot)=e^{A \cdot}$ is a uniformly continuous semigroup.

(H2) The multivalued map $F: J \times E \rightarrow \mathscr{P}_{b, c l, c v}(E)$ satisfies the following:

(2a) For every $t \in J, F(t, \cdot): E \rightarrow \mathscr{P}_{b, c l, c v}$ is u.s.c., for each $x \in E$, the function $F(\cdot, x): J \rightarrow \mathscr{P}_{b, c l, c v}$ is strongly measurable. The set $S_{F, x}=\left\{f \in L^{1}\right.$ $(J, E): f(t) \in F(t, x(t))$, for almost everywhere $t \in J\}$ is nonempty; (2b) There exists a function $\xi_{1} \in L^{1 / p_{1}}\left(J, \mathbb{R}^{+}\right)$, $p_{1} \in(0, p)$ and a continuous nondecreasing function $\psi:[0, \infty) \rightarrow(0, \infty)$, such that for any $(t, x) \in J \times E$, we have $\|F(t, x(t))\|=\sup$ $\{\|f(t)\|: f(t) \in F(t, x(t))\} \leq \xi_{1}(t) \psi\left(\|x\|_{Y}\right)$, $\lim _{m \rightarrow \infty} \inf (\psi(m) / m)=\Lambda<\infty$;

(2c) There exists a constant $p_{2} \in(0, p)$ and a function $\xi_{2} \in L^{1 / p_{2}}\left(J^{\prime}, \mathbb{R}^{+}\right)$s.t. $\beta(F(t, Z)) \leq$ $\xi_{2}(t) \beta\left(t^{1-r} Z\right)$ for any countable subset $Z \subset E$.

(H3) (3a) Linear operator $V: L^{2}(J, U) \rightarrow E$ defined by

$$
\begin{aligned}
V u= & a^{1-r}\left[\Phi_{p, r}(A, a) \sum_{i=1}^{n} a_{i} K \int_{0}^{t_{i}} \Phi_{p, p}\left(A, t_{i}-s\right) B u(s) d s\right. \\
& \left.+\int_{0}^{a} \Phi_{p, p}(A, a-s) B u(s) d s\right]
\end{aligned}
$$

is reversible, the inverse operator denoted by $V^{-1}$ and takes values in $L^{2}(J, U)$ ker $V$, and there exist two constants $N_{u}>0, N_{v}>0$ such that

$$
\|B\| \leq N_{u},\left\|V^{-1}\right\| \leq N_{v}
$$

(3b) There exists a constant $p_{3} \in(0, p)$ and $\xi_{3} \in$ $L^{1 / p_{3}}\left(J^{\prime}, \mathbb{R}^{+}\right)$such that

$$
\beta\left(V^{-1}(Z)(t)\right) \leq \xi_{3}(t) \beta(Z), t \in J,
$$

for any countable subset $Z \subset E$.

(H4) $\lim _{\eta \rightarrow \infty} \sup \eta a^{r-1}\left(N_{p}\left\|x_{1}\right\|+N_{1} N_{r}\left(N_{p}+1\right) \psi(\eta)\right)^{-1}$ $>1$, where $N_{r}=\Gamma(r) N / \Gamma(p)\left(\Gamma(r)-N t_{1}^{r-1} \sum_{i=1}^{n}\left|a_{i}\right|\right)$, $N_{p}=N_{u} N_{v} N_{r}\left(a^{1-q(1-p)} / p\left(a^{1-q(1-p)} / p\right)\right)$ and $d_{i}=b_{i}$ $a^{p-p_{i}}, b_{i}=\left(1-p_{i} / p-p_{i}\right)^{1-p_{i}}, N_{i}=d_{i}\left\|\xi_{i}(s)\right\|_{L^{1 / p_{i}}}, i=$ $1,2,3$.

For any $x \in B_{\gamma}^{Y}\left(J^{\prime}\right)$, define an operator $\mathbb{T}$ as follows:

$$
\begin{aligned}
(\mathbb{} x)(t)= & \Phi_{p, r}(A, t) \sum_{i=1}^{n} a_{i} K \int_{0}^{t_{i}} \Phi_{p, p}\left(A, t_{i}-s\right)[f(s)+B u(s)] d s \\
& +\int_{0}^{t} \Phi_{p, p}(A, t-s)[f(s)+B u(s)] d s, t \in J^{\prime},
\end{aligned}
$$

where $f \in S_{F, x}$.

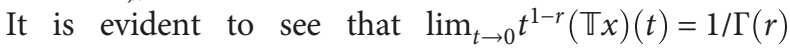
$\sum_{i=1}^{n} a_{i} x\left(t_{i}\right)$.

For any $y \in B_{\gamma}(J)$, let $x(t)=t^{r-1} y(t)$ for $t \in J^{\prime}$, then $x \in B_{\gamma}^{Y}\left(J^{\prime}\right)$. Define $\mathscr{T}$ as follows 


$$
(\mathscr{T} y)(t)= \begin{cases}t^{1-r}(\mathbb{T} x)(t), & t \in J^{\prime} \\ \frac{1}{\Gamma(r)} \sum_{i=1}^{n} a_{i} x\left(t_{i}\right), & t=0\end{cases}
$$

Clearly, $x$ is a mild solution of (3) in $Y$ if and only if $y=\mathscr{T} y$ has a solution $y \in C(J, E)$.

$$
\begin{aligned}
u(t ; x)= & a^{1-r} V^{-1}\left[x_{1}-\Phi_{p, r}(A, a) \sum_{i=1}^{n} a_{i} K \int_{0}^{t_{i}} \Phi_{p, p}\left(A, t_{i}-s\right) f(s) d s\right. \\
& \left.-\int_{0}^{a} \Phi_{p, p}(A, a-s) f(s) d s\right](t), t \in J, f \in S_{F, x} .
\end{aligned}
$$

For brevity, let us take the following notations

$$
\begin{aligned}
\mathscr{P}(t ; x) & =B u(t ; x)+f(t), f \in S_{F, x} ; \\
\tilde{\mathscr{P}}(x) & =\sum_{i=1}^{n} a_{i} K \int_{0}^{t_{i}} \Phi_{p, p}\left(A, t_{i}-s\right) \mathscr{P}(s ; x) d s .
\end{aligned}
$$

In view of Lemma 2, we obtain the following lemma that will be useful in the proof of the main results.

Lemma 7. Under the hypothesis (H2) (2b), (H3) (3a), for each $y \in B_{\gamma}(J)$, set $x(t)=t^{r-1} y(t), t \in J^{\prime}$, we have

$$
\begin{aligned}
\|\mathscr{P}(t ; x)\| \leq & N_{u} N_{v} a^{1-r}\left(\left\|x_{1}\right\|+N_{1} N_{r} \psi(\|y\|)\right) \\
& +\xi_{1}(t) \psi(\|y\|),\|\tilde{\mathscr{T}}(x)\| \leq N_{p} \sum_{i=1}^{n}\left|a_{i}\right|\left\|x_{1}\right\| \\
& +N_{1} N_{r}\left(N_{p}+1\right) \sum_{i=1}^{n}\left|a_{i}\right| \psi(\|y\|) .
\end{aligned}
$$

Proof 2. By Lemma 2, for each $y \in B_{\gamma}(J)$, set $(t)=t^{r-1} y(t)$, $t \in J^{\prime}$, it is easy to get

$$
\begin{aligned}
\|B u(t ; x)\| \leq & N_{u} N_{v}\left[a^{1-r}\left\|x_{1}\right\|+\frac{N^{2}\|K\| \psi\left(\|x\|_{Y}\right) \sum_{i=1}^{n}\left|a_{i}\right|}{\Gamma(r) \Gamma(p)}\right. \\
& \cdot\left\|\int_{0}^{t_{i}}\left(t_{i}-s\right)^{p-1} \xi_{1}(s) d s\right\| \\
& \left.+\frac{a^{1-r} N \psi\left(\|x\|_{Y}\right)}{\Gamma(p)}\left\|\int_{0}^{a}(a-s)^{p-1} \xi_{1}(s) d s\right\|\right] \\
\leq & N_{u} N_{v}\left[a^{1-r}\left\|x_{1}\right\|+\frac{N^{2} \psi(\|y\|) \sum_{i=1}^{n}\left|a_{i}\right| d_{1}\left\|\xi_{1}\right\|_{L^{1 / p_{1}}}}{\Gamma(p)\left(\Gamma(r)-N t_{1}^{r-1} \sum_{i=1}^{n}\left|a_{i}\right|\right)}\right. \\
& \left.+\frac{N d_{1} a^{1-r} \psi(\|y\|)}{\Gamma(p)}\left\|\xi_{1}\right\|_{L^{1 / p_{1}}}\right] \\
\leq & N_{u} N_{v} a^{1-r}\left[\left\|x_{1}\right\|+\frac{\Gamma(r) N N_{1}}{\Gamma(p)\left(\Gamma(r)-N t_{1}^{r-1} \sum_{i=1}^{n}\left|a_{i}\right|\right)} \psi(\|y\|)\right] \\
\triangleq & N_{u} N_{v} a^{1-r}\left(\left\|x_{1}\right\|+N_{1} N_{r} \psi(\|y\|)\right) .
\end{aligned}
$$

Hence, we easily see that

$$
\begin{aligned}
\|\mathscr{P}(t ; x)\| \leq & N_{u} N_{v} a^{1-r}\left(\left\|x_{1}\right\|+N_{1} N_{r} \psi(\|y\|)\right) \\
& +\xi_{1}(t) \psi(\|y\|) ; \\
\|\tilde{\mathscr{P}}(x)\| \leq & \frac{N \Gamma(r) \sum_{i=1}^{n}\left|a_{i}\right|}{\Gamma(p)\left(\Gamma(r)-N t_{1}^{r-1} \sum_{i=1}^{n}\left|a_{i}\right|\right)} \\
& \cdot \int_{0}^{t_{i}}\left(t_{i}-s\right)^{p-1}\|\mathscr{P}(s ; x)\| d s \\
\leq & \frac{N \Gamma(r) \sum_{i=1}^{n}\left|a_{i}\right|}{\Gamma(p)\left(\Gamma(r)-N t_{1}^{r-1} \sum_{i=1}^{n}\left|a_{i}\right|\right)} \\
& \cdot \int_{0}^{t_{i}}\left(t_{i}-s\right)^{p-1}\left[N_{u} N_{v} a^{1-r}\left(\left\|x_{1}\right\|+N_{1} N_{r} \psi(\|y\|)\right)\right. \\
& \left.+\xi_{1}(s) \psi(\|y\|)\right] d s \\
\leq & \frac{\Gamma(r) N N_{u} N_{v} a^{1-q(1-p)} \sum_{i=1}^{n}\left|a_{i}\right|}{\Gamma(p+1)\left(\Gamma(r)-N t_{1}^{r-1} \sum_{i=1}^{n}\left|a_{i}\right|\right)} \\
& \cdot\left(\left\|x_{1}\right\|+N_{1} N_{r} \psi(\|y\|)\right)+N_{1} N_{r} \sum_{i=1}^{n}\left|a_{i}\right| \psi(\|y\|) \\
\triangleq & N_{p} \sum_{i=1}^{n}\left|a_{i}\right|\left\|x_{1}\right\|+N N_{1} N_{r}\left(N_{p}+1\right) \sum_{i=1}^{n}\left|a_{i}\right| \psi(\|y\|) .
\end{aligned}
$$

This completes the proof.

Next, we derive the controllability results for the Hilfer fractional nonlocal differential inclusions (3).

Theorem 1. Assume the hypotheses (HO)-(H4) hold, then the Hilfer fractional nonlocal differential inclusions (3) are exact controllable on J provided that

$$
\Lambda \triangleq N N_{2}\left(1+N_{3} N_{r} N_{u} a^{1-r}\right)\left(\frac{N_{r}}{\Gamma(r)} \sum_{i=1}^{n}\left|a_{i}\right|+\frac{1}{\Gamma(p)}\right)<1
$$

Proof 3. According to (H2) (2a) and [22], for each $x \in C$ $\left(J^{\prime}, E\right)$, the multivalued function $t \rightarrow F(t, x(t))$ has a measurable selection, and in view of (H2) (2b), this selection belongs to $S_{F, x}$. Thus, we can define a multivalued function $\mathscr{T}^{\prime}: C(J, E) \rightarrow 2^{C(J, E)}$ as follows. For every $y \in C(J, E)$, let $x(t)=t^{r-1} y(t) \in Y, t \in J^{\prime}$, and a function $z \in \mathscr{T}^{\prime} y$ if and only if

$$
z(t)=\left\{\begin{array}{l}
K_{r}(t) \sum_{i=1}^{n} a_{i} K \bar{f}\left(t_{i}\right)+t^{1-r} \bar{f}(t), t \in J^{\prime}, \\
\frac{1}{\Gamma(r)} \sum_{i=1}^{n} a_{i} x\left(t_{i}\right), t=0 .
\end{array}\right.
$$

where $K_{r}(t)=t^{1-r} \Phi_{p, r}(A, t), \bar{f}(t)=\int_{0}^{t}(t-s)^{p-1} K_{p}(t-s)(B u$ $(s)+f(s)) d s$ and $f \in S_{F, x}$.

Note that according to (H4), there is $R^{*}$ such that for all $\mathscr{R}>R^{*}$,

$$
a^{1-r}\left[N_{p}\left\|x_{1}\right\|+N_{1} N_{r}\left(N_{p}+1\right) \psi(\mathscr{R})\right]<\mathscr{R} .
$$


Denote $\Omega=\left\{y \in C(J, E):\|y\|_{\infty}<R_{0}\right\}$ and $D=\{y \in C$ (J,E): $\left.\|y\|_{\infty} \leq R_{0}\right\}$, where $R_{0} \geq \gamma$ and $R_{0}=R^{*}+1$. We just prove that the multivalued operator mathcal $T^{\prime}: D \rightarrow 2^{C(J, E)}$ meets the conditions of Lemma 5. Obviously, since the values of $F$ are convex, the values of $\mathscr{T}^{\prime}$ are also convex.
Claim 1. Each solution to the inclusion

$$
y \in \lambda \mathscr{T}^{\prime}(y), y \in D, \lambda \in(0,1)
$$

satisfies $y \notin D-\Omega$.

Let $y$ be a solution of (44). Then, by reminding the definition of $\mathscr{T}^{\prime}$, the hypothesis (H2) (2b) and recalling also Lemma 2, for each $t \in J$, we derive

$$
\begin{aligned}
\|y(t)\| \leq & \frac{N^{2} \sum_{i=1}^{n}\left|a_{i}\right|}{\Gamma(p)\left(\Gamma(r)-N t_{1}^{r-1} \sum_{i=1}^{n}\left|a_{i}\right|\right)} \int_{0}^{t_{i}}\left(t_{i}-s\right)^{p-1}\|\mathscr{P}(s ; x)\| d s+\frac{N t^{1-r}}{\Gamma(p)} \cdot \int_{0}^{t}(t-s)^{p-1}\|\mathscr{P}(s ; x)\| d s \\
\leq & \frac{N^{2} N_{u} N_{v} a^{p+1-r} \sum_{i=1}^{n}\left|a_{i}\right|}{p \Gamma(p)\left(\Gamma(r)-N t_{1}^{r-1} \sum_{i=1}^{n}\left|a_{i}\right|\right)}\left(\left\|x_{1}\right\|+N_{1} N_{r} \psi(\|y\|)\right)+\frac{N N_{u} N_{v} a^{p+2-2 r}}{p \Gamma(p)} \cdot\left(\left\|x_{1}\right\|+N_{1} N_{r} \psi(\|y\|)\right) \\
& +\frac{N a^{1-r} \psi(\|y\|)}{\Gamma(p)} \int_{0}^{t}(t-s)^{p-1} \xi_{1}(s) d s+\frac{N^{2} \psi(\|y\|) \sum_{i=1}^{n}\left|a_{i}\right|}{\Gamma(p)\left(\Gamma(r)-N t_{1}^{r-1} \sum_{i=1}^{n}\left|a_{i}\right|\right)} \int_{0}^{t_{i}}\left(t_{i}-s\right)^{p-1} \xi_{1}(s) d s \\
\leq & \left\{\frac{N^{2} N_{u} N_{v} a^{p+1-r} \sum_{i=1}^{n}\left|a_{i}\right|}{p \Gamma(p)\left(\Gamma(r)-N t_{1}^{r-1} \sum_{i=1}^{n}\left|a_{i}\right|\right)}+\frac{N N_{u} N_{v} \Gamma(r) a^{p+2-2 r}-N^{2} N_{u} N_{v} a^{p+2-2 r} t_{1}^{r-1} \sum_{i=1}^{n}\left|a_{i}\right|}{p \Gamma(p)\left(\Gamma(r)-N t_{1}^{r-1} \sum_{i=1}^{n}\left|a_{i}\right|\right)}\right\} \cdot\left(\left\|x_{1}\right\|+N_{1} N_{r} \psi(\|y\|)\right) \\
& +\left\{\frac{N a^{1-r} \psi(\|y\|) \Gamma(r)-N^{2} a^{1-r} t_{1}^{r-1} \psi(\|y\|) \sum_{i=1}^{n}\left|a_{i}\right|}{\Gamma(p)\left(\Gamma(r)-N t_{1}^{r-1} \sum_{i=1}^{n}\left|a_{i}\right|\right)}+\frac{N^{2} \psi(\|y\|) \sum_{i=1}^{n}\left|a_{i}\right|}{\Gamma(p)\left(\Gamma(r)-N t_{1}^{r-1} \sum_{i=1}^{n}\left|a_{i}\right|\right)}\right\} \cdot d_{1}\left\|\xi_{1}\right\|_{L^{1 / p_{1}}} \\
\leq & \frac{\Gamma(r) N N_{u} N_{v} a^{\mathrm{p}+2-2 r}}{\Gamma(p+1)\left(\Gamma(r)-N t_{1}^{r-1} \sum_{i=1}^{n}\left|a_{i}\right|\right)}\left(\left\|x_{1}\right\|+N_{1} N_{r} \psi(\|y\|)\right)+\frac{\Gamma(r) a^{1-r} N d_{1}\left\|\xi_{1}\right\|_{L^{1 / p_{1}}} \psi(\|y\|)}{\Gamma(p)\left(\Gamma(r)-N t_{1}^{r-1} \sum_{i=1}^{n}\left|a_{i}\right|\right)} \\
\leq & \frac{\Gamma(r) N N_{u} N_{v} a^{p+2-2 r}\left\|x_{1}\right\|}{\Gamma(p+1)\left(\Gamma(r)-N t_{1}^{r-1} \sum_{i=1}^{n}\left|a_{i}\right|\right)}+a^{1-r} N_{1} N_{r} \psi\left(R_{0}\right)\left[\frac{\Gamma(r) N N_{u} N_{v} a^{p+1-r}}{\Gamma(p+1)\left(\Gamma(r)-N t_{1}^{r-1} \sum_{i=1}^{n}\left|a_{i}\right|\right)}+1\right] \\
\triangleq & a^{1-r}\left[N_{p}\left\|x_{1}\right\|+N_{1} N_{r}\left(N_{p}+1\right) \psi\left(R_{0}\right)\right] .
\end{aligned}
$$

This inequality with (43) deduces $\|y\|_{\infty}<R_{0}$, So $y \notin D-\Omega$.

Claim 2. Every function in $\left\{\mathscr{T}^{\prime} y: y \in B_{\gamma}(J)\right\}$ is equicontinuous.
For each $y \in B_{\gamma}(J)$, set $x(t)=t^{r-1} y(t), t \in J^{\prime}$ such that $z \in \mathscr{T}^{\prime}(y)$. By (42), there is $f \in S_{F, x}$, for $\tau_{1}, \tau_{2} \in J, \tau_{1}<\tau_{2}$, we get

$$
\begin{aligned}
\left\|z\left(\tau_{2}\right)-z\left(\tau_{1}\right)\right\| \leq & \left\|K_{r}\left(\tau_{2}\right) \tilde{\mathscr{P}}(x)-K_{r}\left(\tau_{1}\right) \tilde{\mathscr{P}}(x)\right\| \\
& +\left|\tau_{2}^{1-r} \int_{0}^{\tau_{2}}\left(\tau_{2}-s\right)^{p-1} K_{p}\left(\tau_{2}-s\right) \mathscr{P}(s ; x) d s-\tau_{1}^{1-r} \int_{0}^{\tau_{1}}\left(\tau_{1}-s\right)^{p-1} K_{p}\left(\tau_{1}-s\right) \mathscr{P}(s ; x) d s\right| \\
\leq & \left\|K_{r}\left(\tau_{2}\right)-K_{r}\left(\tau_{1}\right)\right\||| \tilde{\mathscr{P}}(x) \|+\left|\int_{\tau_{1}}^{\tau_{2}} \tau_{2}^{1-r}\left(\tau_{2}-s\right)^{p-1} K_{p}\left(\tau_{2}-s\right) \mathscr{P}(s ; x) d s\right| \\
& +\left|\int_{0}^{\tau_{1}} \tau_{1}^{1-r}\left(\tau_{1}-s\right)^{p-1}\left[K_{p}\left(\tau_{2}-s\right)-K_{p}\left(\tau_{1}-s\right)\right] \mathscr{P}(s ; x) d s\right| \\
& +\left|\int_{0}^{\tau_{1}}\left[\tau_{1}^{1-r}\left(\tau_{1}-s\right)^{p-1}-\tau_{2}^{1-r}\left(\tau_{2}-s\right)^{p-1}\right] K_{p}\left(\tau_{2}-s\right) \mathscr{P}(s ; x) d s\right| \\
\leq & I_{1}+I_{2}+I_{3}+I_{4},
\end{aligned}
$$


where

$$
\begin{aligned}
& I_{1}=\left\|K_{r}\left(\tau_{2}\right)-K_{r}\left(\tau_{1}\right)\right\||| \tilde{\mathscr{P}}(x)||, \\
& I_{2}=\frac{N}{\Gamma(p)}\left|\int_{\tau_{1}}^{\tau_{2}} \tau_{2}^{1-r}\left(\tau_{2}-s\right)^{p-1} \mathscr{P}(s ; x) d s\right|, \\
& I_{3}=\frac{N}{\Gamma(p)}\left|\int_{0}^{\tau_{1}}\left[\tau_{1}^{1-r}\left(\tau_{1}-s\right)^{p-1}-\tau_{2}^{1-r}\left(\tau_{2}-s\right)^{p-1}\right] \mathscr{P}(s ; x) d s\right|, \\
& I_{4}=\left|\int_{0}^{\tau_{1}} \tau_{1}^{1-r}\left(\tau_{1}-s\right)^{p-1}\left[K_{p}\left(\tau_{2}-s\right)-K_{p}\left(\tau_{1}-s\right)\right] \mathscr{P}(s ; x) d s\right| .
\end{aligned}
$$

From (H1), one can deduce that $I_{1} \rightarrow 0$ as $\tau_{2}-\tau_{1} \rightarrow 0$. From Lemma 7, we have

$$
\begin{aligned}
I_{2} \leq & \frac{N N_{u} N_{v} a^{2-2 r}}{\Gamma(p+1)}\left(\left\|x_{1}\right\|+N_{1} N_{r} \psi(\gamma)\right)\left(\tau_{2}-\tau_{1}\right)^{p} \\
& +\frac{N b_{1} a^{1-r}}{\Gamma(p)}\left(\tau_{2}-\tau_{1}\right)^{p-p_{1}}\left\|\xi_{1}\right\|_{L^{1 / p_{1}}} \psi(\gamma),
\end{aligned}
$$

which implies that $I_{2} \rightarrow 0$ as $\tau_{2}-\tau_{1} \rightarrow 0$.

$$
\begin{aligned}
I_{3} \leq & \frac{N N_{u} N_{v} a^{1-r}}{\Gamma(p)}\left(\left\|x_{1}\right\|+N_{1} N_{r} \psi(\gamma)\right) \\
& \cdot \int_{0}^{\tau_{1}}\left[\tau_{1}^{1-r}\left(\tau_{1}-s\right)^{p-1}-\tau_{2}^{1-r}\left(\tau_{2}-s\right)^{p-1}\right] d s \\
& +\frac{N}{\Gamma(p)} \psi(\gamma) \int_{0}^{\tau_{1}}\left[\tau_{1}^{1-r}\left(\tau_{1}-s\right)^{p-1}-\tau_{2}^{1-r}\left(\tau_{2}-s\right)^{p-1}\right] \xi_{1}(s) d s .
\end{aligned}
$$

Noting that

$$
\left[\tau_{1}^{1-r}\left(\tau_{1}-s\right)^{p-1}-\tau_{2}^{1-r}\left(\tau_{2}-s\right)^{p-1}\right] \xi_{1}(s) \leq \tau_{1}^{1-r}\left(\tau_{1}-s\right)^{p-1} \xi_{1}(s)
$$

and $\int_{0}^{\tau_{1}} \tau_{1}^{1-r}\left(\tau_{1}-s\right)^{p-1} \xi_{1}(s) d s, s \in\left[0, \tau_{1}\right]$ exists, then by Lebesgue's dominated convergence theorem, we obtain $I_{3} \rightarrow 0$ as $\tau_{2}-\tau_{1} \rightarrow 0$.

For $\varepsilon>0$, we get

$$
\begin{aligned}
I_{4} \leq & \int_{0}^{\tau_{1}-\varepsilon} \tau_{1}^{1-r}\left(\tau_{1}-s\right)^{p-1}\left\|\mathrm{~K}_{p}\left(\tau_{2}-s\right)-K_{p}\left(\tau_{1}-s\right)\right\|\|\mathscr{P}(s ; x)\| d s \\
& +\int_{\tau_{1}-\varepsilon}^{\tau_{1}} \tau_{1}^{1-r}\left(\tau_{1}-s\right)^{p-1}\left\|K_{p}\left(\tau_{2}-s\right)-K_{p}\left(\tau_{1}-s\right)\right\|\|\mathscr{P}(s ; x)\| d s \\
\leq & N_{u} N_{v} a^{2-2 r}\left(\left\|x_{1}\right\|+N_{1} N_{r} \psi(\gamma)\right) \frac{\tau_{1}^{p}-\varepsilon^{p}}{p} \sup _{s \in\left[0, \tau_{1}-\varepsilon\right]}\left\|K_{p}\left(\tau_{2}-s\right)-K_{p}\left(\tau_{1}-s\right)\right\| \\
& +\tau_{1}^{1-r} \int_{0}^{\tau_{1}-\varepsilon}\left(\tau_{1}-s\right)^{p-1} \xi_{1}(s) d s \sup _{s \in\left[0, \tau_{1}-\varepsilon\right]}\left\|K_{p}\left(\tau_{2}-s\right)-K_{p}\left(\tau_{1}-s\right)\right\| \psi(\gamma) \\
& +\frac{2 N N_{u} N_{v} a^{2-2 r} \varepsilon^{p}}{\Gamma(p+1)}\left(\left\|x_{1}\right\|+N_{1} N_{r} \psi(\gamma)\right)+\frac{2 N a^{1-r}}{\Gamma(p)} b_{1} \varepsilon^{p-p_{1}} \psi(\gamma)\left\|\xi_{1}\right\|_{L^{1 / p_{1}}} .
\end{aligned}
$$

The assumption (H1) guarantees that $I_{4} \rightarrow 0$ as $\tau_{2}-$ $\tau_{1} \rightarrow 0$ and $\varepsilon \rightarrow 0$. We prove that $K_{r}(t)$ is uniformly continuous on $J$. Consequently, $\mathscr{T}^{\prime}$ is equicontinuous on $B_{\gamma}(J)$.

Claim 3. The inference (13) holds with $y_{0}=0$.

Let $Z=\operatorname{conv}\left(\left\{y_{0}\right\} \cup \mathscr{T}^{\prime}(Z)\right) \subseteq D, \bar{Z}=\bar{G}$ with $G \subseteq Z$ countable. We assert that $Z$ is relatively compact. In fact, since $G$ is countable and $G \subseteq Z=\operatorname{conv}\left(\left\{y_{0}\right\} \cup \mathscr{T}^{\prime}(Z)\right)$, we can chase down a countable set $\mathscr{H}=\left\{z_{n}: n \geq 1\right\} \subseteq \mathscr{T}^{\prime}(Z)$ with $G \subseteq \operatorname{conv}\left(\left\{y_{0}\right\} \cup \mathscr{H}\right)$. Then, there exists $y_{n} \in Z$ with $z_{n} \in \mathscr{T}^{\prime}\left(y_{n}\right)$. This means that there is $f_{n} \in S_{F, x_{n}}$ such that for $t \in J$

$$
z_{n}(t)=K_{r}(t) \sum_{i=1}^{n} a_{i} K\left(\bar{f}_{n}\left(t_{i}\right)\right)+\bar{f}_{n}(t)
$$

where

$$
\bar{f}_{n}(t)=\int_{0}^{t}(t-s)^{p-1} K_{p}(t-s)\left[B u_{n}(s)+f_{n}(s)\right] d s
$$

From $Z \subseteq \bar{G} \subseteq \overline{\operatorname{conv}}\left(\left\{y_{0}\right\} \cup \mathscr{H}\right)$, for $t \in J, s \in[0, t]$. By utilizing Lemmas 2, 4, (H2) (2c), (H3) (3b), and the properties of the noncompact measure, we can derive 


$$
\begin{aligned}
\beta\left(B u_{n}(s)+f_{n}(s)\right) \leq & N_{u} \xi_{3}(s)\left\{\frac{N^{2} \sum_{i=1}^{n}\left|a_{i}\right|}{\Gamma(p)\left(\Gamma(r)-N t_{1}^{r-1} \sum_{i=1}^{n}\left|a_{i}\right|\right)} \int_{0}^{t_{i}}\left(t_{i}-s\right)^{p-1} \beta\left\{f_{n}(s): n \geq 1\right\} d s\right. \\
& \left.+\frac{N a^{1-r}}{\Gamma(p)} \int_{0}^{a}(a-s)^{p-1} \beta\left\{f_{n}(s): n \geq 1\right\} d s\right\}+\beta\left\{f_{n}(s): n \geq 1\right\} \\
\leq & N_{u} \xi_{3}(s)\left\{\frac{N^{2} \sum_{i=1}^{n}\left|a_{i}\right|}{\Gamma(p)\left(\Gamma(r)-N t_{1}^{r-1} \sum_{i=1}^{n}\left|a_{i}\right|\right)} \int_{0}^{t_{i}}\left(t_{i}-s\right)^{p-1} \xi_{2}(s) \beta\left\{y_{n}(s): n \geq 1\right\} d s\right. \\
& \left.+\frac{N a^{1-r}}{\Gamma(p)} \int_{0}^{a}(a-s)^{p-1} \xi_{2}(s) \beta\left\{y_{n}(s): n \geq 1\right\} d s\right\}+\xi_{2}(s) \beta\left\{y_{n}(s): n \geq 1\right\} \\
\leq & {\left[N_{2} N_{u} N_{r} a^{1-r} \xi_{3}(s)+\xi_{2}(s)\right] \cdot \beta(Z), }
\end{aligned}
$$

and

$$
\begin{aligned}
\beta(Z(t)) \leq & \beta(\bar{G}(t)) \leq \beta(\mathscr{H}(t)) \\
\leq & \beta\left\{K_{r}(t) \sum_{i=1}^{n} a_{i} K \int_{0}^{t_{i}}\left(t_{i}-s\right)^{p-1} K_{p}\left(t_{i}-s\right)\right. \\
& \left.\cdot\left[B u_{n}(s)+f_{n}(s)\right] d s: n \geq 1\right\} \\
& +\beta\left\{\int_{0}^{t}(t-s)^{p-1} K_{p}(t-s)\left[B u_{n}(s)+f_{n}(s)\right] d s: n \geq 1\right\} \\
\leq & \frac{N^{2} \sum_{i=1}^{n}\left|a_{i}\right|}{\Gamma(p)\left(\Gamma(r)-N t_{1}^{r-1} \sum_{i=1}^{n}\left|a_{i}\right|\right)} \\
& \cdot \int_{0}^{t_{i}}\left(t_{i}-s\right)^{p-1}\left[N_{2} N_{u} N_{r} a^{1-r} \xi_{3}(s)+\xi_{2}(s)\right] d s \cdot \beta(Z) \\
& +\frac{N}{\Gamma(p)} \int_{0}^{t}(t-s)^{p-1}\left[N_{2} N_{u} N_{r} a^{1-r} \xi_{3}(s)+\xi_{2}(s)\right] d s \cdot \beta(Z) \\
\leq & N N_{2}\left(1+N_{3} N_{r} N_{u} a^{1-r}\right)\left(\frac{N_{r}}{\Gamma(r)} \sum_{i=1}^{n}\left|a_{i}\right|+\frac{1}{\Gamma(p)}\right) \cdot \beta(Z) \\
\triangleq & \Lambda \cdot \beta(Z) .
\end{aligned}
$$

Reminding $Z=\operatorname{conv}\left(\left\{y_{0}\right\} \cup \mathscr{T}^{\prime}(Z)\right)$, by Claim $2, Z$ is equicontinuous. So we find from Lemma 3 that

$$
\beta(Z)=\max _{t \in J} \beta(Z(t)) \leq \Lambda \beta(Z) .
$$

Since $\Lambda<1$, we obtain $\beta(Z)=0$. That is, $\bar{Z}$ is compact.

Claim 4. $\mathscr{T}^{\prime}$ maps compact sets into relatively compact sets. Let $Q$ be a compact subset of $Z$. From Claim 2, $\mathscr{T}^{\prime}(Q)$ is equicontinuous. Let $t \in J$, by the definition of $\mathscr{T}^{\prime}$, for any $y \in Q$ and $z \in \mathscr{T}^{\prime}(y)$, there is $f_{y} \in S_{F, x}$ such that

$$
z(t)=K_{r}(t) \sum_{i=1}^{n} a_{i} K\left(\bar{f}_{y}\left(t_{i}\right)\right)+\bar{f}_{y}(t), t \in J
$$

where $\bar{f}_{y}(t)=\int_{0}^{t}(t-s)^{p-1} K_{p}(t-s)\left(B u_{y}(s)+f_{y}(s)\right) d s$.

Therefore

$$
\begin{aligned}
\beta\left(\mathscr{T}^{\prime}(Q)(t)\right) \leq & \beta\left\{z(t): z \in \mathscr{T}^{\prime}(y), y \in Q\right\} \\
\leq & \beta\left\{K_{r}(t) \sum_{i=1}^{n} a_{i} K \int_{0}^{t_{i}}\left(t_{i}-s\right)^{p-1} K_{p}\left(t_{i}-s\right)\right. \\
& \left.\cdot\left[B u_{y}(s)+f_{y}(s)\right] d s: y \in G\right\} \\
& +\beta\left\{\int_{0}^{t}(t-s)^{p-1} K_{p}(t-s)\right. \\
& \left.\cdot\left[B u_{y}(s)+f_{y}(s)\right] d s: y \in G\right\} \\
\leq & N N_{2}\left(1+N_{3} N_{r} N_{u} a^{1-r}\right) \\
& \cdot\left(\frac{N_{r}}{\Gamma(r)} \sum_{i=1}^{n}\left|a_{i}\right|+\frac{1}{\Gamma(p)}\right) \beta(Q)=0 .
\end{aligned}
$$

Then, Lemma 3 indicates $\beta\left(\mathscr{T}^{\prime}(Q)\right)=\max _{t \in J} \beta\left(\mathscr{T}^{\prime}(Q)\right.$ $(t))=0$, that is, the set $\mathscr{T}^{\prime}(Q)$ is relatively compact.

Claim 5. The graph $\mathscr{T}^{\prime}$ is closed.

For any $y^{(n)} \in B_{\gamma}(J)$, let $x^{(n)}(t)=t^{r-1} y^{(n)}(t)$ and $x^{*}$ $(t)=t^{r-1} y^{*}(t), t \in J^{\prime}$. Let $y^{(n)} \rightarrow y^{*}(n \rightarrow \infty), \mu^{(n)} \in \mathscr{T}^{\prime}\left(y^{(n)}\right)$, $\mu^{(n)} \rightarrow \mu^{*}(n \rightarrow \infty)$. We will show that $\mu^{*} \in \mathscr{T}^{\prime}\left(y^{*}\right)$. Since $\mu^{(n)} \in \mathscr{T}^{\prime}\left(y^{(n)}\right)$, there is $f^{(n)} \in S_{F, x^{(n)}}$ such that for any $t \in J$, 


$$
\begin{aligned}
\mu^{(n)}(t)= & K_{r}(t) \sum_{i=1}^{n} a_{i} K \int_{0}^{t_{i}}\left(t_{i}-s\right)^{p-1} K_{p}\left(t_{i}-s\right) f^{(n)}(s) d s \\
& +K_{r}(t) \sum_{i=1}^{n} a_{i} K \int_{0}^{t_{i}}\left(t_{i}-s\right)^{p-1} \cdot K_{p}\left(t_{i}-s\right) B V^{-1} \\
& \cdot\left[a^{1-r} x_{1}-K_{r}(a) \sum_{i=1}^{n} a_{i} K\right. \\
& \cdot \int_{0}^{t_{i}}\left(t_{i}-\tau\right)^{p-1} K_{p}\left(t_{i}-\tau\right) f^{(n)}(\tau) d \tau \\
& \left.-a^{1-r} \int_{0}^{a}(a-\tau)^{p-1} K_{p}(a-\tau) f^{(n)}(\tau) d \tau\right] d s \\
& +t^{1-r} \int_{0}^{t}(t-s)^{p-1} K_{p}(t-s) f^{(n)}(s) d s \\
& +t^{1-r} \int_{0}^{t}(t-s)^{p-1} K_{p}(t-s) B V^{-1} \\
& \cdot\left[a^{1-r} x_{1}-K_{r}(s) \sum_{i=1}^{n} a_{i} K\right. \\
& \cdot \int_{0}^{t_{i}}\left(t_{i}-\tau\right)^{p-1} \cdot K_{p}\left(t_{i}-\tau\right) f^{(n)}(\tau) d \tau-a^{1-r} \\
& \left.\cdot \int_{0}^{a}(a-\tau)^{p-1} K_{p}(a-\tau) f^{(n)}(\tau) d \tau\right] d s .
\end{aligned}
$$

So we just need to demonstrate the existence of $f^{*} \in S_{F, x^{*}}$ such that for any $t \in J$,

$$
\begin{aligned}
\mu^{*}(t)= & K_{r}(t) \sum_{i=1}^{n} a_{i} K \int_{0}^{t_{i}}\left(t_{i}-s\right)^{p-1} K_{p}\left(t_{i}-s\right) f^{*}(s) d s \\
& +K_{r}(t) \sum_{i=1}^{n} a_{i} K \int_{0}^{t_{i}}\left(t_{i}-s\right)^{p-1} \cdot K_{p}\left(t_{i}-s\right) B V^{-1} \\
& \cdot\left[a^{1-r} x_{1}-K_{r}(a) \sum_{i=1}^{n} a_{i} K \int_{0}^{t_{i}}\left(t_{i}-\tau\right)^{p-1} K_{p}\left(t_{i}-\tau\right) f^{*}(\tau) d \tau\right. \\
& \left.-a^{1-r} \int_{0}^{a}(a-\tau)^{p-1} K_{p}(a-\tau) f^{*}(\tau) d \tau\right] d s \\
& +t^{1-r} \int_{0}^{t}(t-s)^{p-1} K_{p}(t-s) \cdot f^{*}(s) d s \\
& +t^{1-r} \int_{0}^{t}(t-s)^{p-1} K_{p}(t-s) B V^{-1} \\
& \cdot\left[a^{1-r} x_{1}-K_{r}(s) \sum_{i=1}^{n} a_{i} K \cdot \int_{0}^{t_{i}}\left(t_{i}-\tau\right)^{p-1} K_{p}\left(t_{i}-\tau\right) f^{*}(\tau) d \tau\right. \\
& \left.-a^{1-r} \int_{0}^{a}(a-\tau)^{p-1} K_{p}(a-\tau) \mathrm{f}^{*}(\tau) d \tau\right] d s .
\end{aligned}
$$

$$
\begin{aligned}
\| \mu^{(n)}(t) & -K_{r}(t) \sum_{i=1}^{n} a_{i} K \int_{0}^{t_{i}}\left(t_{i}-s\right)^{p-1} K_{p}\left(t_{i}-s\right) B V^{-1} a^{1-r} x_{1} d s \\
& -t^{1-r} \int_{0}^{t}(t-s)^{p-1} \cdot K_{p}(t-s) B V^{-1} a^{1-r} x_{1} d s \\
& -\left[\mu^{*}(t)-K_{r}(t) \sum_{i=1}^{n} a_{i} K \int_{0}^{t_{i}}\left(t_{i}-s\right)^{p-1} K_{p}\left(t_{i}-s\right)\right. \\
& \cdot B V^{-1} a^{1-r} x_{1} d s-t^{1-r} \\
& \left.\cdot \int_{0}^{t}(t-s)^{p-1} K_{p}(t-s) B V^{-1} a^{1-r} x_{1} d s\right] \| \rightarrow 0 .
\end{aligned}
$$

In addition, we get

$$
\begin{aligned}
\mu^{(n)}(t)- & K_{r}(t) \sum_{i=1}^{n} a_{i} K \int_{0}^{t_{i}}\left(t_{i}-s\right)^{p-1} K_{p}\left(t_{i}-s\right) B V^{-1} a^{1-r} x_{1} d s \\
& -t^{1-r} \int_{0}^{t}(t-s)^{p-1} K_{p}(t-s) B V^{-1} a^{1-r} x_{1} d s \in \Gamma\left(S_{F, x^{(n)}}\right) .
\end{aligned}
$$

Take into account the linear continuous operator

$$
\Gamma: L^{1 / p}(J, E) \rightarrow B_{\gamma}(J)
$$

Clearly, we can get from Lemma 1 that the operator $\Gamma \circ S_{F}$ closed graph.

Since $\mu^{(n)} \rightarrow \mu^{*}, n \rightarrow \infty$, we can obtain that as $n \rightarrow \infty$,

$$
\begin{aligned}
(\Gamma f)(t)= & K_{r}(t) \sum_{i=1}^{n} a_{i} K \int_{0}^{t_{i}}\left(t_{i}-s\right)^{p-1} K_{p}\left(t_{i}-s\right) \\
& \cdot\left\{f(s)-B V^{-1}\left[K_{r}(s) \sum_{i=1}^{n} a_{i} K\right.\right. \\
& \cdot \int_{0}^{t_{i}}\left(t_{i}-\tau\right)^{p-1} K_{p}\left(t_{i}-\tau\right) f(\tau) d \tau+a^{1-r} \\
& \left.\left.\cdot \int_{0}^{a}(a-\tau)^{p-1} K_{p}(a-\tau) f(\tau) d \tau\right]\right\} d s \\
& +t^{1-r} \int_{0}^{t}(t-s)^{p-1} K_{p}(t-s) \\
& \cdot\left\{f(s)-B V^{-1} \cdot\left[K_{r}(s) \sum_{i=1}^{n} a_{i} K\right.\right. \\
& \cdot \int_{0}^{t_{i}}\left(t_{i}-\tau\right)^{p-1} \cdot K_{p}\left(t_{i}-\tau\right) f(\tau) d \tau \\
& \left.\left.+a^{1-r} \int_{0}^{a}(a-\tau)^{p-1} K_{p}(a-\tau) f(\tau) d \tau\right]\right\} d s .
\end{aligned}
$$




$$
\begin{aligned}
\mu^{*}(t)- & K_{r}(t) \sum_{i=1}^{n} a_{i} K \int_{0}^{t_{i}}\left(t_{i}-s\right)^{p-1} K_{p}\left(t_{i}-s\right) B V^{-1} a^{1-r} x_{1} d s \\
& -t^{1-r} \int_{0}^{t}(t-s)^{p-1} K_{p}(t-s) B V^{-1} a^{1-r} x_{1} d s \\
= & K_{r}(t) \sum_{i=1}^{n} a_{i} K \int_{0}^{t_{i}}\left(t_{i}-s\right)^{p-1} K_{p}\left(t_{i}-s\right) f^{*}(s) d s \\
& -K_{r}(t) \sum_{i=1}^{n} a_{i} K \int_{0}^{t_{i}}\left(t_{i}-s\right)^{p-1} \cdot K_{p}\left(t_{i}-s\right) B V^{-1} \\
& \cdot\left[K_{r}(s) \sum_{i=1}^{n} a_{i} K \int_{0}^{t_{i}}\left(t_{i}-\tau\right)^{p-1} K_{p}\left(t_{i}-\tau\right) f^{*}(\tau) d \tau+a^{1-r}\right. \\
& \left.\cdot \int_{0}^{a}(a-\tau)^{p-1} K_{p}(a-\tau) f^{*}(\tau) d \tau\right] d s \\
& +t^{1-r} \int_{0}^{t}(t-s)^{p-1} K_{p}(t-s) f^{*}(s) d s \\
& -t^{1-r} \int_{0}^{t}(t-s)^{p-1} K_{p}(t-s) B V^{-1} \\
& \cdot\left[K_{r}(s) \sum_{i=1}^{n} a_{i} K \int_{0}^{t_{i}}\left(t_{i}-\tau\right)^{p-1} K_{p}\left(t_{i}-\tau\right) \cdot f^{*}(\tau) d \tau\right. \\
& \left.+a^{1-r} \int_{0}^{a}(a-\tau)^{p-1} K_{p}(a-\tau) f^{*}(\tau) d \tau\right] d s .
\end{aligned}
$$

For some $f^{*} \in S_{F, x^{*}}$, this infers that $\mu^{*} \in \mathscr{T}^{\prime}\left(y^{*}\right)$. Hence, $\mathscr{T}^{\prime}$ has a closed graph. Thus, Claims 1-5 are completed. By use of Lemma 5, we know operator $\mathscr{T}^{\prime}$ has a fixed point in $B_{\gamma}(J)$. Let $x(t)=t^{r-1} y(t)$, then $x$ is a mild solution of (3) and it satisfies $x(a)=x_{1}$. Therefore, the Hilfer fractional nonlocal differential inclusions (3) are exact controllable on $J$.

\section{Applications}

Consider the following partial differential system

$$
\begin{aligned}
D_{0^{+}}^{3 / 4,2 / 3} x(t, s) \in & -\frac{\partial}{\partial s} x(t, s) \\
& +\frac{e^{-2 t}}{1+e^{t}} x(t, s)+\eta(t, s), \quad t \in(0, a], s \in(0,1), \\
x(t, 0)= & x(t, 1)=0, \quad t \in(0, a], \\
\left.I_{0^{+}}^{1 / 3(1-p)} x(t, s)\right|_{t=0}= & \sum_{i=1}^{n} \Gamma\left(\frac{11}{12}\right) \arctan \frac{1}{2 i^{2}} x\left(t_{i}, s\right), \quad s \in(0,1),
\end{aligned}
$$

where $n \in(0, a)$ is constants, $\eta: J \times(0,1) \rightarrow(0,1)$ is continuous.

Let $E=\Omega=: C([0,1])$ and $A$ is defined by

$$
\begin{aligned}
D(A) & =\left\{w \in E: w^{\prime} \in E, w(0)=w(1)=0\right\}, \\
A w & =-w^{\prime}, w \in D(A) .
\end{aligned}
$$

As we all know that $-A$ generates an equicontinuous semigroup $S(t)(t \geq 0)$ in $E$ and it satisfies

$$
T(t) w(v)=w(t+v)
$$

for $w \in E$. Thus, $S(t)(t \geq 0)$ is not compact in $E$ and $\sup _{0 \leq t \leq a}\|T(t)\| \leq 1$. Take

$$
\begin{aligned}
x(t)(s) & =x(t, s), \\
D_{0^{+}}^{3 / 4,2 / 3} x(t)(s) & =I_{0^{+}}^{1 / 6} D_{0^{+}}^{11 / 12} x(t, s) \\
& =\frac{1}{\Gamma(1 / 6)} \int_{0}^{t}(t-\tau)^{-5 / 6} \frac{\partial^{11 / 12}}{\partial \tau^{11 / 12}} x(\tau, s) d \tau, \\
f(t, x(t))(s) & =\frac{e^{-2 t}}{1+e^{t}} x(t, s), \\
u(t)(s) & =\mu(t, s), \\
a_{i} & =\Gamma\left(\frac{11}{12}\right) \arctan \frac{1}{2 i^{2}}, t_{i}=i, i=1,2, \ldots, n .
\end{aligned}
$$

Then, for any $y \in B_{\gamma}:=\{y \in C(J, E):\|y\| \leq \gamma\}$, where $J=$ $[0, \mathrm{a}]$, let $x(t)=t^{-(1 / 12)} y(t), t \in J^{\prime}:=(0, a]$, then $x \in B_{\gamma}^{Y}\left(J^{\prime}\right)$, and we obtain

$$
\|f(t, x(t))(s)\| \leq \frac{e^{-2 t}}{1+e^{t}}\|x(t, s)\| \leq \frac{e^{-2 t} \gamma}{1+e^{t}} \leq \frac{\gamma}{2} .
$$

Thus, the hypothesis $(H 2)$ holds for $\beta=1 / 2$ and $\xi_{2}(t)=$ $1 / 2$ for all $t \in J^{\prime}$. By

$$
\sum_{i=1}^{n}\left|a_{i}\right| \leq \Gamma\left(\frac{11}{12}\right) \cdot \sum_{i=1}^{\infty} \arctan \frac{1}{2 i^{2}}=\Gamma\left(\frac{11}{12}\right) \cdot \frac{\pi}{4}<\Gamma\left(\frac{11}{12}\right),
$$

we verify that the hypothesis $(\mathrm{H} 0)$ holds.

For $s \in(0,1)$, the operator $V$ is defined as

$$
\begin{aligned}
(V(t))(s)= & K_{r}(a)\left[I-\Gamma\left(\frac{11}{12}\right) \sum_{i=1}^{n} i^{-(1 / 12)} K_{r}(i) \arctan \frac{1}{2 i^{2}}\right]^{-1} \\
& \cdot \Gamma\left(\frac{11}{12}\right) \sum_{i=1}^{n} \arctan \frac{1}{2 i^{2}} \\
& \cdot \int_{0}^{i}(i-\tau)^{-(1 / 4)} K_{p}(i-\tau) \eta(\tau, s) d \tau \\
& +\int_{0}^{a}(a-\tau)^{-(1 / 4)} K_{p}(a-\tau) \eta(\tau, s) d \tau
\end{aligned}
$$

where $\left\{K_{r}(t)\right\}_{(t \geq 0)}$ and $\left\{K_{p}(t)\right\}_{(t \geq 0)}$ satisfy

$$
\begin{aligned}
& K_{r}(t)=I_{0^{+}}^{1 / 6}\left(t^{-(1 / 4)} K_{p}(t)\right), \\
& K_{p}(t)=\sum_{i=0}^{\infty} \frac{A^{i} t^{(3 / 4) i}}{\Gamma((3 / 4) i+(3 / 4))} .
\end{aligned}
$$


If $V$ satisfies the hypothesis (H3), from Theorem 1, we get that the Hilfer fractional differential inclusion (66) involving nonlocal initial conditions is exact controllable on $[0, a]$ provided that (H4) and (41) are satisfied.

\section{Data Availability}

The data used to support the findings of this study are included within the article.

\section{Conflicts of Interest}

The authors declare that they have no conflicts of interest.

\section{Acknowledgments}

This work was supported by the National Natural Science Foundation of China (nos. 1371027, 11471015, and 1601003), Natural Science Foundation of Anhui Province (no. 1708085MA15), and Natural Science Fund of Colleges and Universities in Anhui Province (no. KJ2018A0470).

\section{References}

[1] K. Diethelm and A. D. Freed, "On the solution of nonlinear fractional-order differential equations used in the modeling of viscoplasticity," in Scientific Computing in Chemical Engineering II-Computational Fluid Dynamics, Reaction Engineering and Molecular Properties, F. Keil, W. Mackens, H. Voss, and J. Werther, Eds., pp. 217-224, Springer-Verlag, Heidelberg, 1999.

[2] A. A. Kilbas, H. M. Srivastava, and J. J. Trujillo, Theory and Applications of Fractional Differential Equations. NorthHolland and Mathematics Studies, Elsevier Science BV, Netherlands Amsterdam, 2006.

[3] K. S. Miller and B. Ross, An Introduction to the Fractional Calculus and Fractional Differential Equations, Wiley, New York, NY, USA, 1993.

[4] I. Podlubny, Fractional Differential Equations, Academic Press, San Diego, CA, USA, 1999.

[5] Y. Zhou, Basic Theory of Fractional Differential Equations, World Scientific, Singapore, 2014.

[6] M. M. el-Borai, "Some probability densities and fundamental solutions of fractional evolution equations," Chaos Solitons Fractals, vol. 14, no. 3, pp. 433-440, 2002.

[7] Y. Zhou and F. Jiao, "Existence of mild solutions for fractional neutral evolution equations," Computers and Mathematics with Applications, vol. 59, no. 3, pp. 1063-1077, 2010.

[8] S. Liu, X. Wu, X. F. Zhou, and W. Jiang, "Asymptotical stability of Riemann-Liouville fractional nonlinear systems," Nonlinear Dynamics, vol. 86, no. 1, pp. 65-71, 2016.

[9] S. Liu, X. F. Zhou, X. Y. Li, and W. Jiang, "Asymptotical stability of Riemann-Liouville fractional singular systems with multiple time-varying delays," Applied Mathematics Letters, vol. 65, pp. 32-39, 2017.

[10] K. X. Li, J. G. Peng, and J. X. Jia, "Cauchy problems for fractional differential equations with Riemann-Liouville fractional derivatives," Journal of Functional Analysis, vol. 263, no. 2, pp. 476-510, 2012.

[11] R. P. Agarwal and A. Özbekler, "Lyapunov type inequalities for mixed nonlinear Riemann-Liouville fractional differential equations with a forcing term," Journal of Computational and Applied Mathematics, vol. 314, pp. 69-78, 2017.

[12] R. P. Agarwal, V. Lupulescu, D. O’Regan, and G. ur Rahman, "Fractional calculus and fractional differential equations in nonreflexive Banach spaces," Communications in Nonlinear Science Numerical Simulation, vol. 20, no. 1, pp. 59-73, 2015.

[13] H. Liu, Y. Pan, S. Li, and Y. Chen, "Adaptive fuzzy backstepping control of fractional-order nonlinear systems," IEEE Transactions on Systems, Man, and Cybernetics: Systems, vol. 47, no. 8, pp. 2209-2217, 2017.

[14] H. Liu, S. Li, J. Cao, G. Li, A. Alsaedi, and F. E. Alsaadi, "Adaptive fuzzy prescribed performance controller design for a class of uncertain fractional-order nonlinear systems with external disturbances," Neurocomputing, vol. 219, pp. 422430, 2017.

[15] H. Liu, S. Li, G. Li, and H. Wang, "Adaptive controller design for a class of uncertain fractional-order nonlinear systems: an adaptive fuzzy approach," International Journal of Fuzzy Systems, vol. 20, no. 2, pp. 366-379, 2018.

[16] R. Hilfer, Applications of Fractional Calculus in Physics, World Scientific, Singapore, 2000.

[17] R. Hilfer, Y. Luchko, and Ž. Tomovski, "Operational method for the solution of fractional differential equations with generalized Riemann-Liouville fractional derivatives," Fractional Calculus and Applied Analysis, vol. 12, pp. 289-318, 2009.

[18] K. M. Furati, M. D. Kassim, and N. E. Tatar, "Existence and uniqueness for a problem involving Hilfer fractional derivative," Computers and Mathematics with Applications, vol. 64, no. 6, pp. 1616-1626, 2012.

[19] H. B. Gu and J. J. Trujillo, "Existence of mild solution for evolution equation with Hilfer fractional derivative," Applied Mathematics and Computation, vol. 257, pp. 344354, 2015.

[20] M. Eduardo Hernández and D. O’Regan, "Controllability of Volterra-Fredholm type systems in Banach spaces," Journal of the Franklin Institute, vol. 346, no. 2, pp. 95-101, 2009.

[21] S. C. Ji, G. Li, and M. Wang, "Controllability of impulsive differential systems with nonlocal conditions," Applied Mathematics and Computation, vol. 217, no. 16, pp. 69816989, 2011.

[22] Y.-K. Chang, A. Anguraj, and M. Mallika Arjunan, "Controllability of impulsive neutral functional differential inclusions with infinite delay in Banach spaces," Chaos Solitons Fractals, vol. 39, no. 4, pp. 1864-1876, 2009.

[23] V. Obukhovski and P. Zecca, "Controllability for systems governed by semilinear differential inclusions in a Banach space with a noncompact semigroup," Nonlinear Analysis, vol. 70, no. 9, pp. 3424-3436, 2009.

[24] N. Abada, M. Benchohra, and H. Hammouche, "Existence and controllability results for nondensely defined impulsive semilinear functional differential inclusions," Journal of Differential Equations, vol. 246, no. 10, pp. 3834-3863, 2009.

[25] X.-F. Zhou, J. Wei, and L.-G. Hu, "Controllability of a fractional linear time-invariant neutral dynamical system," Applied Mathematics Letters, vol. 26, no. 4, pp. 418-424, 2013.

[26] J. Wei, "The controllability of fractional control systems with control delay," Computers and Mathematics with Applications, vol. 64, no. 10, pp. 3153-3159, 2012.

[27] K. Balachandran, S. Divya, L. Rodríguez-Germá, and J. J. Trujillo, "Relative controllability of nonlinear neutral fractional integro-differential systems with distributed delays in 
control," Mathematical Methods in the Applied Sciences, vol. 39, no. 2, pp. 214-224, 2016.

[28] N. I. Mahmudov and S. Zorlu, "On the approximate controllability of fractional evolution equations with compact analytic semigroup," Journal of Computational and Applied Mathematics, vol. 259, pp. 194-204, 2014.

[29] Z. Liu and X. Li, “Approximate controllability of fractional evolution systems with Riemann-Liouville fractional derivatives," SIAM Journal on Control and Optimization, vol. 53, no. 4, pp. 1920-1933, 2015.

[30] M. Yang and Q. R. Wang, "Approximate controllability of Riemann-Liouville fractional differential inclusions," Applied Mathematics and Computation, vol. 274, pp. 267-281, 2016.

[31] M. Yang and Q. R. Wang, "Approximate controllability of Hilfer fractional differential inclusions with nonlocal conditions," Mathematical Methods in the Applied Sciences, vol. 40, no. 4, pp. 1126-1138, 2017.

[32] J. R. Wang and Y. Zhou, "Complete controllability of fractional evolution systems," Communications in Nonlinear Science and Numerical Simulation, vol. 17, no. 11, pp. 43464355, 2012.

[33] J. Liang and H. Yang, "Controllability of fractional integrodifferential evolution equations with nonlocal conditions," Applied Mathematics and Computation, vol. 254, pp. 2029, 2015.

[34] L. Byszewski and V. Lakshmikantham, "Theorem about the existence and uniqueness of a solution of a nonlocal abstract Cauchy problem in a Banach space," Applicable Analysis, vol. 40 , no. 1, pp. 11-19, 1991.

[35] L. Byszewski, "Theorems about the existence and uniqueness of solutions of a semilinear evolution nonlocal Cauchy problem," Journal of Mathematical Analysis and Applications, vol. 162, no. 2, pp. 494-505, 1991.

[36] A. Boucherif and R. Precup, "Semilinear evolution equations with nonlocal initial conditions," Dynamic Systems and Applications, vol. 16, no. 3, pp. 507-516, 2007.

[37] Q. Liu, "Existence results for a class of semilinear nonlocal evolution equations," Mathematical Methods in the Applied Sciences, vol. 35, no. 11, pp. 1356-1364, 2012.

[38] Y. Zhou and F. Jiao, "Nonlocal Cauchy problem for fractional evolution equations," Nonlinear Analysis: Real World Applications, vol. 11, no. 5, pp. 4465-4475, 2010.

[39] F. Li, J. Liang, and H.-K. Xu, "Existence of mild solutions for fractional integrodifferential equations of Sobolev type with nonlocal conditions," Journal of Mathematical Analysis and Applications, vol. 391, no. 2, pp. 510-525, 2012.

[40] A. Chauhan and J. Dabas, "Local and global existence of mild solution to an impulsive fractional functional integrodifferential equation with nonlocal condition," Communications in Nonlinear Science and Numerical Simulation, vol. 19, no. 4, pp. 821-829, 2014.

[41] J. R. Wang, A. G. Ibrahim, and M. Fečkan, "Nonlocal impulsive fractional differential inclusions with fractional sectorial operators on Banach spaces," Applied Mathematics and Computation, vol. 257, pp. 103-118, 2015.

[42] J. R. Wang, A. G. Ibrahim, and M. Fečkan, "Nonlocal Cauchy problems for semilinear differential inclusions with fractional order in Banach spaces," Communications in Nonlinear Science and Numerical Simulation, vol. 27, no. 1-3, pp. 281-293, 2015.
[43] J. Du, W. Jiang, D. Pang, and A. U. K. Niazi, “Controllability for a new class of fractional neutral integro-differential evolution equations with infinite delay and nonlocal conditions," Advances in Difference Equations, vol. 2017, no. 1, 2017.

[44] A. Lasota and Z. Opial, "An application of the KakutaniKy-Fan theorem in the theory of ordinary differential equations," Bulletin of Academy of Polish Sciences, Serial Sciences of Mathematics Astronomy Physics, vol. 13, pp. 781-786, 1965.

[45] M. Kamenskii, V. V. Obukhovskii, and P. Zecca, Condensing Multivalued Maps and Semilinear Differential Inclusions in Banach Spaces, Walter de Gruyter, Berlin, NY, USA, 2001.

[46] J. Banaś, "On measures of noncompactness in Banach spaces," Commentationes Mathematicae Universitatis Carolinae, vol. 21, no. 1, pp. 131-143, 1980.

[47] D. O'Regan and R. Precup, "Fixed point theorems for setvalued maps and existence principles for integral inclusions," Journal of Mathematical Analysis and Applications, vol. 245, no. 2, pp. 594-612, 2000.

[48] J. R. Wang, Y. Zhou, and M. Fečkan, “Alternative results and robustness for fractional evolution equations with periodic boundary conditions," Electronic Journal of Qualitative Theory of Differential Equations, no. 97, pp. 1-15, 2011. 


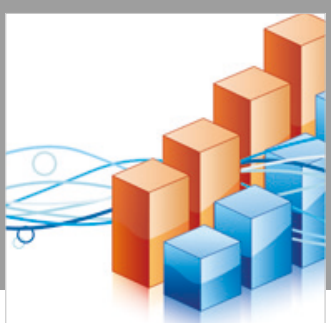

Advances in

Operations Research

\section{-n-m}
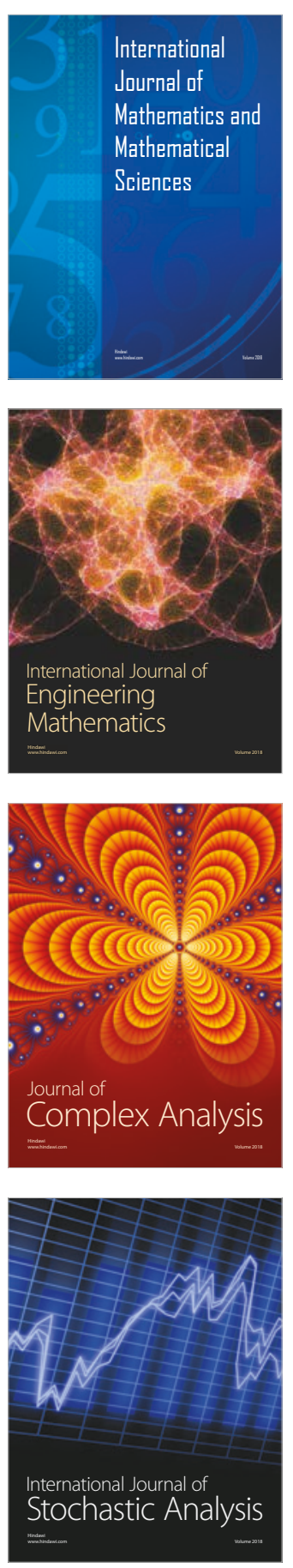
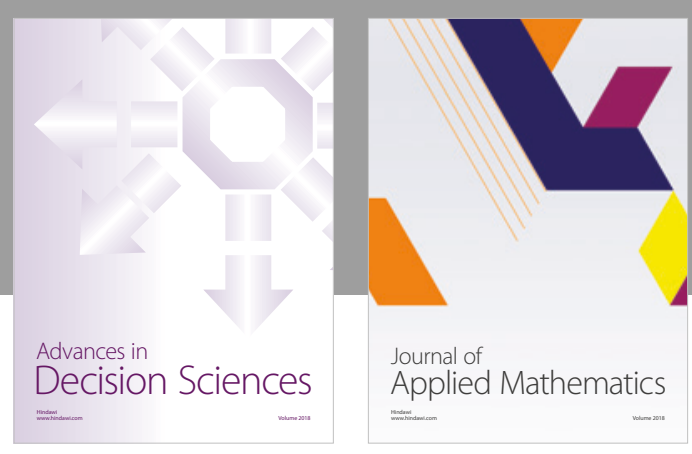

Journal of

Applied Mathematics
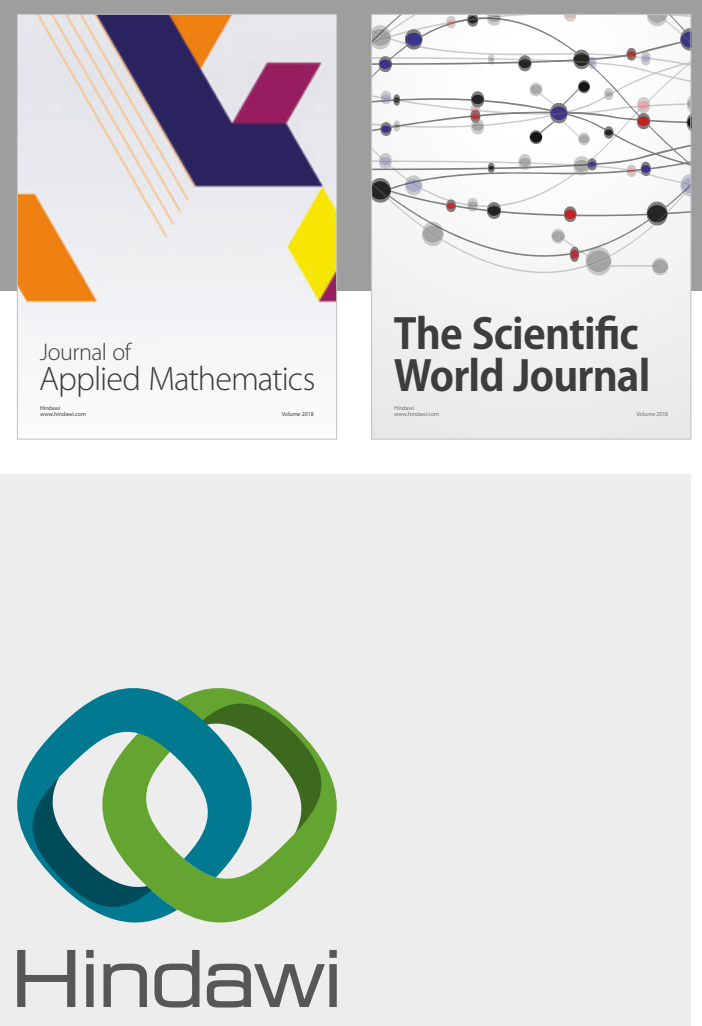

Submit your manuscripts at

www.hindawi.com

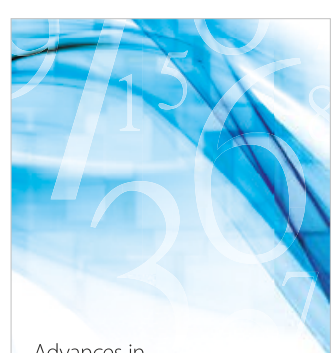

Advances in
Numerical Analysis
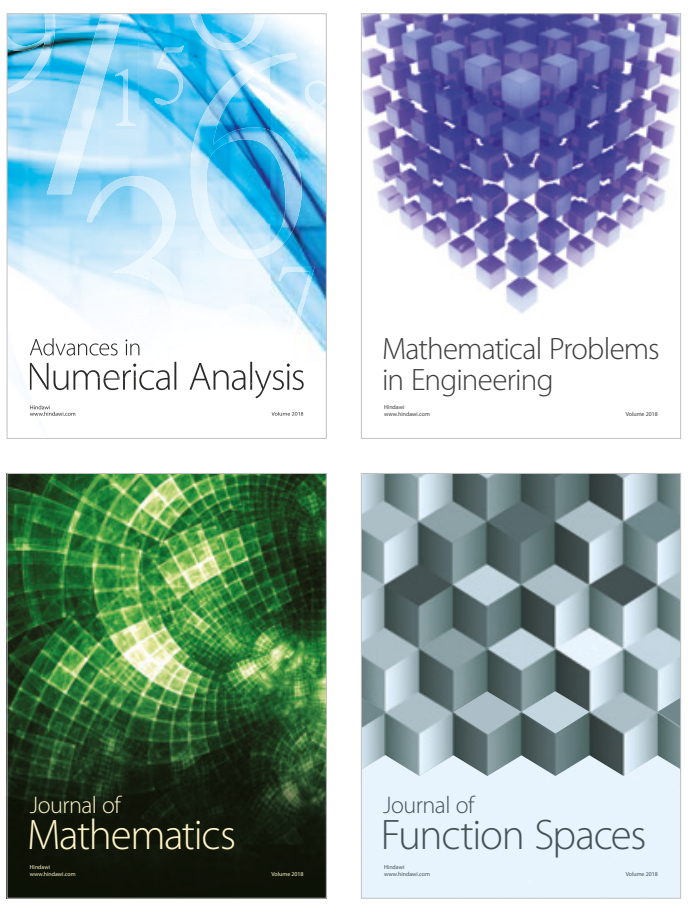

Mathematical Problems in Engineering

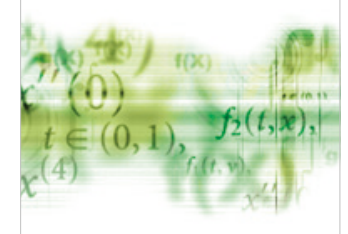

International Journal of

Differential Equations

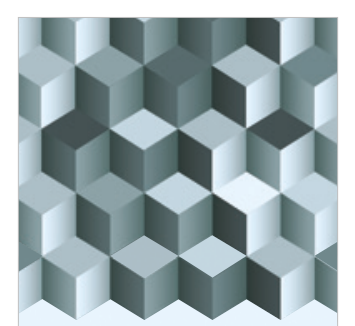

Journal of

Function Spaces
The Scientific

World Journal

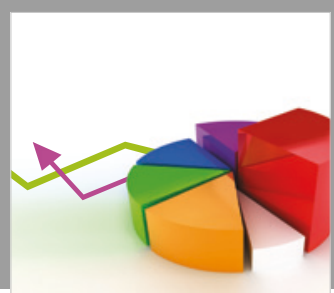

Journal of

Probability and Statistics
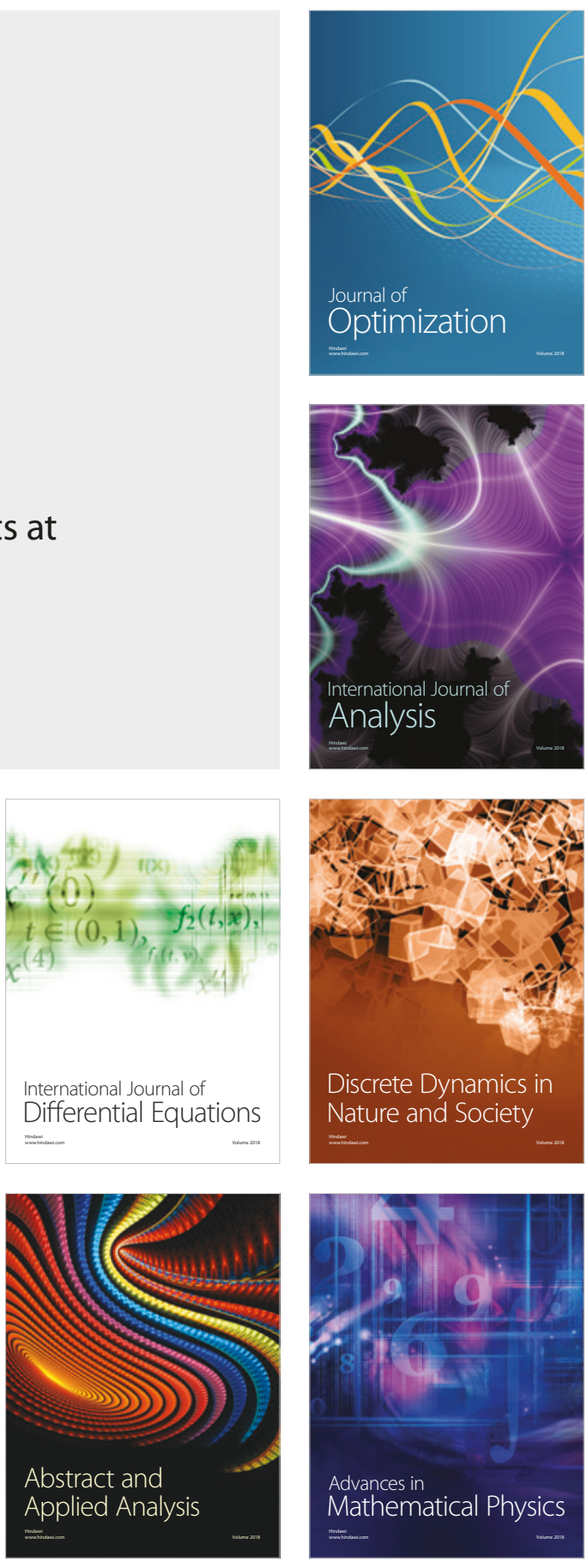